
$\sum$ Research Square
Preprints are preliminary reports that have not undergone peer review.
They should not be considered conclusive, used to inform clinical practice,
or referenced by the media as validated information.

\title{
Systematic analysis of rare variants in mitochondrial function-associated genes for autosomal-dominant Parkinson's disease in a Chinese population
}

Yongping Chen

Sichuan University West China Hospital

Xiaojing Gu

Sichuan University West China Hospital

Ruwei Ou

Sichuan University West China Hospital

Lingyu Zhang

Sichuan University West China Hospital

\section{Yanbing Hou}

Sichuan University West China Hospital

\section{Kuncheng Liu}

Sichuan University West China Hospital

\section{Bei Cao}

Sichuan University West China Hospital

Qianqian Wei

Sichuan University West China Hospital

Wei Song

Sichuan University West China Hospital

Bi Zhao

Sichuan University West China Hospital

Ying Wu

Sichuan University West China Hospital

Jingqiu Cheng

Sichuan University West China Hospital

huifang shang ( $\nabla$ hfshang2002@126.com)

Sichuan University West China Hospital Department of Neurology https://orcid.org/0000-0003-0947-1151

Research article

Keywords: Parkinson's disease, autosomal dominant, mitochondrial function-associated genes, HTRA2, CHCHD2, burden analysis

Posted Date: April 28th, 2020

DOI: https://doi.org/10.21203/rs.3.rs-23120/v1

License: (1) This work is licensed under a Creative Commons Attribution 4.0 International License. Read Full License 


\section{Abstract \\ Background}

Mitochondrial dysfunction is involved in the pathogenicity of Parkinson's disease (PD). However, the genetic roles of mitochondrial function-associated genes responsible for PD need to be replicated in different cohorts.

\section{Methods}

Whole-exome and Sanger sequencing were used to identify the genetic etiology of 400 autosomal dominant-inherited PD (ADPD) patients. Variants in six dominant inherited mitochondrial function-associated genes, including HTRA2, CHCHD2, CHCHD10, TRAP1, HSPA9 and RHOT1, were analyzed.

\section{Results}

A total of 12 rare variants identified in the five genes accounted for $3 \%$ of ADPD cases, including $0.5 \%$ in $H$ TRA2, $0.8 \%$ in $C H C H D 2,1 \%$ in $T R A P 1,0.3 \%$ in RHOT1 and 0.5\% in HSPA9. Among them, five novel variants, p.E4A, p.R13Cfs*107 and p.R449X in TRAP1, p.S95N in RHOT1 and p.N180I in HSPA9, were

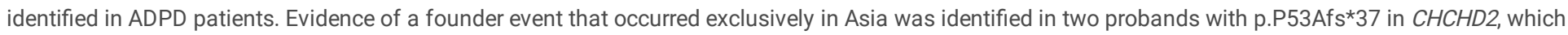
was further observed in one patient from 300 sporadic cases. Based on burden analysis, $C H C H D 2$ tended to be slightly enriched in ADPD. Clinically, all patients carrying mutations in the genes presented typical motor symptoms and a good response to L-DOPA. Most of them had slower disease progression (8/12) and mild cognitive impairment (9/12), but the age of onset varied. No rare variant was detected in $\mathrm{CHCHD10}$.

\section{Conclusion}

Our study expands the mutation spectra and enhances the understanding of the clinical phenotype of PD patients with mitochondrial function-related gene variants. Additionally, the $\mathrm{CHCHD} 2$ gene should be given more attention in PD originating in the Chinese population.

\section{Background}

Parkinson's disease (PD), the second most common neurodegenerative disorder secondary to Alzheimer's disease, is characterized by cardinal motor and nonmotor symptoms. The prevalence rises from $1 \%$ in those over 60 years to $4 \%$ in the population over 80 [1]. Loss of dopaminergic neurons in the substantia nigra and Lewy body formation are the pathological hallmarks of PD. However, the molecular mechanisms underlying dopaminergic neuronal cell death are still not fully understood.

Mitochondrial dysfunction, as one of the disease-associated pathological pathways involved in PD, is a well-established and shared feature between sporadic and monogenic PD patients. In PD patients, mitochondria isolated from platelets and skeletal muscle have been confirmed to have reduced complex I and IV activity[2], and defective specific complexes from the mitochondrial respiratory chain were also identified in postmortem brains of PD patients[3], suggesting an important role of mitochondria in the pathogenesis of the disease. However, the identification of rare monogenic forms of PD and the subsequent functional studies related to disease-causing mutations have substantially advanced our understanding of mitochondrial dysfunction pathways driving neurodegeneration.

To date, several mitochondrial function-associated genes contribute to the development/risk of PD. Among them, three causative genes, parkin (PARK2), PTEN-induced putative kinase 1 (PINK1, PARK6) and DJ-1 (PARK7), are unambiguously linked to autosomal recessive juvenile PD and early-onset PD, and these mutations account for $3-7 \%, 2-4 \%$ and $1-2 \%$ of early-onset PD(EOPD) cases in individuals of European ancestry, respectively[4]. However, mutations in the other three mitochondrial-related genes, high-temperature-regulated A2 (HTRA2, PARK13)[5], coiled-coil-helix-coiled-coil-helix domain containing 2 (CHCHD2, PARK22)[6] and coiled-coil-helix-coiled-coil-helix domain containing 10 (CHCHD10)[7], have been reported to contribute to the development of autosomal dominant PD (ADPD) or other neurodegenerative diseases such as amyotrophic lateral sclerosis (ALS). In addition, two mitochondrial chaperone proteins, tumor necrosis factor receptor associated protein-1 (TRAP1) and mortalin (encoded by heat shock 70 kDa protein 9, HSPA9), could modify the asynuclein toxicity induced by loss of function from the PINK1/parkin pathway[8] or decrease the tolerance of cells to exogenous oxidative stress [9]. Importantly, heterozygous pathogenic variants in TRAP1 and HSPA9 were also found in PD patients [10, 11]. Very recently, two studies on European ancestry found that mutation of ras homolog family member T1 (RHOT1), which encodes another pivotal mitochondrial transport protein (Miro1) and serves as mitochondrial-endoplasmic reticulum (ER) contact sites, also caused ADPD[12,13]. However, the role of the six Mendelian dominantly inherited mitochondrial function-associated genes is still awaiting confirmation or has not been duplicated in PD patients of different ethnicities.

Hence, in this study, based on an isolated and genetically homogeneous Chinese ADPD cohort, we explored the rare variant frequencies of the six mitochondrial function-related genes, the collective burden caused by rare variants in the six genes for PD, and the clinical spectrum of patients with rare variants in the six genes. Additionally, we critically summarized the results for HTRA2 and CHCHD2 reported in previously published studies.

\section{Methods}


A total of 400 patients with ADPD (mean age of onset: $53.4 \pm 6.0$ years old, range from 15.6 to 80.1 years old) admitted to the Department of Neurology, West China Hospital from December 2010 to June 2018 were recruited into the study. These patients were diagnosed by experienced neurologists based on the United Kingdom PD Society Brain Bank Clinical Diagnostic Criteria [14] or 2015 MDS clinical diagnostic criteria[15] for PD. Patients who had at least one firstdegree PD-affected relative were categorized as familial PD (FPD), and patients with at least two consecutive generations of PD in their families were classified as ADPD [16]. Demographic data (including age, sex, dominant hand, education level, height, weight, past history, etc.) and clinical data (including age of onset, disease duration, initial medication, etc.) of all the participants were collected. Unified Parkinson's Disease Rating Scale (UPDRS) part III and H\&Y stage were used to evaluate motor severity. Cognitive function was evaluated by the Montreal Cognitive Assessment (MoCA) and frontal assessment battery (FAB). The study was approved by the ethics committee of Sichuan University, and all the subjects who participated in the study completed informed consent prior to participating.

\section{DNA preparation and genetic analysis}

Genomic DNA was collected from peripheral blood leukocytes via standard phenol-chloroform procedures. Genetic analysis, including WES, MLPA, data analysis, variant annotation, haplotype analysis, dating and sequencing analysis for CHCHD2 p.P53Afs*37 in sporadic PD are described in detail in the Supplementary Methods.

\section{Burden analysis}

The differences in frequency distributions of rare variants of HTRA2, CHCHD2, CHCHD10, TRAP1, HSPA9 and RHOT1 between the GnomAD database and ADPD patients were determined using Fisher's exact test. In this study, rare variants (MAF $\leq 0.1 \%$ ) of the HTRA2, CHCHD2, CHCHD10, TRAP1, HSPA9 and RHOT1 genes were obtained from ADPD by WES as the case group, and rare variants from the East Asian cohort in GnomAD v2.1 (including 9977 individuals) were obtained as the control group. The putative pathogenic variants (PPVs), including deleterious missense variants predicted by Condel and splicing, stopgain, indel, and start-loss variants, were included for burden analysis. Statistical significance was defined as $p<0.05$.

\section{Results}

\section{Demographic characteristics}

The demographic and clinical characteristics of the patients included in the study are presented in Supplementary Table 1. Among all the participating patients, twenty families had PD symptoms throughout three generations, while the remaining families had two consecutive generations affected by PD.

\section{Novel rare variants in six mitochondrial function-related PD genes identified in ADPD}

A total of 12 rare candidate variants in the five genes, which have not been previously reported in PD, were identified in 13 unrelated probands. Furthermore, all 13 patients were confirmed to not carry any pathogenic/likely pathogenic variants in other known PD causative genes based on our comprehensive genetic analysis. Among the variants, there were 2 rare variants in HTRA2, 2 rare variants in CHCHD2 (including one variant in 2 probands), 5 rare variants in TRAP1, 2 variants in HSPA9 and one rare variant in RHOT1. However, no rare variant in $\mathrm{CHCHD10}$ was found. According to ACMG guidelines, 10 variants were classified as pathogenic or likely pathogenic variants, one variant as VUS, and one variant as benign. Among the 12 rare variants, five of them, including p.E4A, p.R13Cfs*107 and p.R449X in TRAP1, p.S95N in RHOT1 and p.N180I in HSPA9 were novel (Table 1) and were not found in the abovementioned public databases. Excluding the benign variant in one patient, the frequency of patients with rare variants in all six genes in our ADPD cohort was $3 \%$ (12/400). 
Table 1

Details of mitochondrial pathogenic variants identified in familial PD patients

\begin{tabular}{|c|c|c|c|c|c|c|c|c|c|}
\hline Pedigree & Genes & $\begin{array}{l}\text { Chromosomal } \\
\text { Position* }\end{array}$ & $\begin{array}{l}\text { cDNA } \\
\text { alteration }\end{array}$ & $\begin{array}{l}\text { Amino Acid } \\
\text { Change }\end{array}$ & $\begin{array}{l}\text { Variant } \\
\text { Type }\end{array}$ & Rs. No & $\begin{array}{l}\text { GnomAD } \\
\text { total }\end{array}$ & $\begin{array}{l}\text { GnomAD } \\
\text { East } \\
\text { Asian }\end{array}$ & $\begin{array}{l}\text { SIFT } \\
\text { score }\end{array}$ \\
\hline 1 & HTRA2 & chr2:74757185 & c. $52 \mathrm{C}>\mathrm{T}$ & R18W & missense & rs866473711 & $1 / 230126$ & $0 / 17588$ & 0 \\
\hline 2 & HTRA2 & chr2:74757948 & c. $711 \mathrm{G}>\mathrm{C}$ & $\mathrm{K} 237 \mathrm{~N}$ & missense & rs773298397 & $1 / 251458$ & 0/18394 & 0.15 \\
\hline $3-4$ & CHCHD2 & chr7:56172062 & c.156_157insGCAG & $\mathrm{P} 53 \mathrm{Afs} * 37$ & frameshift & NA & $1 / 247008$ & $1 / 18338$ & - \\
\hline 5 & $\mathrm{CHCHD2}$ & chr7:56171922 & c.296dupA & Y99X & $\begin{array}{l}\text { stop } \\
\text { gained }\end{array}$ & rs200052223 & $1 / 249888$ & $1 / 18380$ & - \\
\hline 6 & TRAP1 & chr16:3715998 & c.1345_1357del & $\mathrm{R} 449 \mathrm{X}$ & $\begin{array}{l}\text { stop } \\
\text { gained }\end{array}$ & Novel & - & - & - \\
\hline 7 & TRAP1 & chr16:3708138 & c. $2107 \mathrm{C}>\mathrm{T}$ & R703X & $\begin{array}{l}\text { stop } \\
\text { gained }\end{array}$ & rs371020906 & $52 / 282572$ & $7 / 19948$ & - \\
\hline 8 & TRAP1 & chr16:3767473 & c.36_37insTGCTGTGGGGC & $\mathrm{R} 13 \mathrm{Cfs} * 107$ & frameshift & Novel & - & - & - \\
\hline 9 & TRAP1 & chr16:3767499 & $\mathrm{c} .11 \mathrm{~T}>\mathrm{G}$ & E4A & missense & Novel & - & - & 0.01 \\
\hline 10 & TRAP1 & chr16:3740966 & c. $109 \mathrm{G}>\mathrm{A}$ & R37W & missense & rs140670575 & $20 / 219430$ & $2 / 14338$ & 0.15 \\
\hline 11 & RHOT1 & chr17:30503187 & c. $284 \mathrm{G}>\mathrm{A}$ & S95N & missense & Novel & - & - & 0.26 \\
\hline 12 & HSPA9 & chr5:137903408 & c. $539 \mathrm{~T}>\mathrm{A}$ & N180I & missense & Novel & - & - & 0 \\
\hline 13 & HSPA9 & chr5:137909471 & c. $209 A>G$ & M70T & missense & rs971461123 & - & - & 0 \\
\hline
\end{tabular}

* Position on Genome Reference Consortium human genome build 37 (GRCh37); N, neutral; D, deleterious; GERP, Genomic Evolutionary Rate Profiling; CADD, ( College of Medical Genetics and Genomics.

\section{HTRA2}

Two rare missense variants (p.R18W and p.K237W) in HTRA2 were found in two probands (0.5\%). Both of them were classified as likely pathogenic, and the locations of all reported variants in HTRA2 in PD patients are shown in Fig. 1A and Supplementary Table 2. These two patients had late-onset, typical motor symptoms and good response to L-DOPA (Table 2). The patient with the K237N variant developed hyposmia, sleep dysfunction, and mild cognitive impairment in the early stage of the disease. Interestingly, his father at 65 years old, his younger sister at 73 years old, and his younger son at 43 years old were reported to have parkinsonian symptoms (Fig. 1F, family 2). Sanger sequencing confirmed that his younger son carried the K237N variant, but his healthy older son and daughter did not.

Table 2

Clinical features of patients carrying variants in our

\begin{tabular}{|c|c|c|c|c|c|c|c|c|c|c|c|c|}
\hline Family & Gene & Mutation & Sex & OA & Duration & $\begin{array}{l}\text { Initial } \\
\text { symptoms }\end{array}$ & $\begin{array}{l}\text { UPDRS- } \\
\text { III }\end{array}$ & $\begin{array}{l}\text { UPDRS- } \\
\text { III/duration }\end{array}$ & H\&Y & H\&Y/duration & $\begin{array}{l}\text { L-DOPA } \\
\text { responsiveness }\end{array}$ & $\begin{array}{l}\text { Motor } \\
\text { fluctua }\end{array}$ \\
\hline 1 & HTRA2 & R18W & $M$ & 64.61 & 2.98 & tremor & 29 & 9.74 & 2 & 0.67 & Good & - \\
\hline 2 & HTRA2 & $\mathrm{K} 237 \mathrm{~N}$ & $M$ & 67.87 & 8.05 & PIGD & 46 & 5.72 & 2.5 & 0.31 & Good & - \\
\hline 3 & $\mathrm{CHCHD2}$ & P53Afs*37 & $\mathrm{F}$ & 55.36 & 12.68 & tremor & 59 & 4.65 & 2.5 & 0.20 & Good & + \\
\hline 4 & $\mathrm{CHCHD} 2$ & P53Afs*37 & $M$ & 35.83 & 4.01 & tremor & 19 & 4.74 & 2 & 0.50 & Good & - \\
\hline 5 & $\mathrm{CHCHD} 2$ & Y99X & $\mathrm{F}$ & 40.84 & 2.59 & tremor & 68 & 26.29 & 4 & 1.55 & Good & - \\
\hline 6 & TRAP1 & R449X & $M$ & 41.04 & 4.76 & tremor & 30 & 6.30 & 2 & 0.42 & Good & + \\
\hline 8 & TRAP1 & R13Cfs*107 & $M$ & 60.31 & 0.41 & tremor & 7 & 17.26 & 1.5 & 3.70 & Good & - \\
\hline 9 & TRAP1 & $\mathrm{E} 4 \mathrm{~A}$ & $\mathrm{~F}$ & 47.00 & 4.75 & tremor & 26 & 5.48 & 2 & 0.42 & Good & - \\
\hline 10 & TRAP1 & R37W & $\mathrm{F}$ & 59.73 & 1.48 & tremor & 16 & 10.81 & 1.5 & 1.01 & Good & - \\
\hline 11 & RHOT1 & S95N & $M$ & 60.15 & 2.28 & tremor & 29 & 12.72 & 2 & 0.88 & Good & - \\
\hline 12 & HSPA9 & N180I & $\mathrm{F}$ & 44.32 & 2.90 & rigidity & 3 & 1.04 & 1 & 0.35 & Good & + \\
\hline 13 & HSPA9 & M70T & $M$ & 34.65 & 4.31 & tremor & 17 & 3.94 & 2 & 0.46 & Good & - \\
\hline
\end{tabular}

OA, onset age; M, male; F, female; HY, Hoehn-Yahr score; MOCA, Montreal Cognitive Assessment score; FBA, frontal assessment battery score; RBD, Sleep Beh 


\section{CHCHD2}

Two protein-truncating variants (p.P53Afs*37 and p.Y99X), which caused loss of the $\mathrm{CHCH}$ domain (Fig. 1B and Supplementary Table 3), were found in three unrelated patients $(0.75 \%)$ who presented tremor as the initial symptom and mild cognitive impairment even in the early stage of the disease (duration of 2.59 years), but the age of onset varied (ranging from 35.8-55.3 years old) (Table 2). Interestingly, one of the two patients carrying p.P53Afs*37 additionally developed cervical dystonia that progressed to the cranial region over a disease duration of 3.5 years. He had a good response to levodopa for his parkinsonism symptoms but limited relief of dystonia. His mother developed right lower limb resting tremor, right-sided stiffness and bradykinesia at the age of 66 (Fig. 1G, family 4). Sanger sequencing and cosegregation analysis identified that his mother carried the same mutation. Interestingly, the p.P53Afs 37 variant was also reported in one East Asian individual from GnomeAD (1/9169), combined with its high enrichment in our cohort, suggesting a common founder effect. The results of haplotype analysis for genetic polymorphisms containing $\mathrm{CHCHD2}$ found that they shared the same haplotype in the 8.18-Mb interval (Table 4), and genetic mutation age analysis by Gamma estimated that the mutation arose at least 65 generations ago (1300 years ago assuming a 20 -year intergeneration time). These results were consistent with inheritance of the p.P53Afs 37 variant, which was "identical by descent", from a common ancestor in the two unrelated patients. To further define the role of $\mathrm{CHCHD} 2 \mathrm{p} . \mathrm{P} 53 \mathrm{Afs} \mathrm{s}^{*} \mathrm{3}$ in PD from our cohort, it was scanned in 300 sporadic PD patients, and we identified one patient whose onset age was 44.53 years carried the same variant, but not found in 300 controls.

Table 4

Haplotypes of genetic markers surrounding $\mathrm{CHCHD} 2$ in patients harboring P53Afs*37 mutation

\begin{tabular}{|lllllll|}
\hline SNPs & Position & Refer & Frequency & Family 3 & Family 4 & Share \\
\hline rs34016595 & 56007576 & C & 0.29 & TT & CC & - \\
\hline rs199535022 & 56087278 & C & $1.67 E-5$ & CC & AC & C \\
\hline rs6593297 & 56122058 & A & 0.69 & AT & TT & T \\
\hline rs2230197 & 56126360 & T & 0.69 & TC & CC & C \\
\hline rs10800 & 56147589 & A & 0.24 & AA & GA & A \\
\hline rs199778139 & 56149477 & A & 0.05 & AC & AC & C \\
\hline rs13238521 & 56149939 & G & 0.08 & GA & GA & A \\
\hline rs2242510 & 56151816 & C & 0.05 & CG & CG & G \\
\hline P53Afs*37 & 56172062 & - & - & - & - & \\
\hline rs816407 & 56174140 & G & 0.83 & TT & TT & T \\
\hline$\ldots \ldots .$. & $\ldots \ldots$ & & & & & \\
\hline rs12536582 & 56899910 & A & 0.08 & CA & CA & A \\
\hline rs376138445 & 56900733 & C & 0.39 & CC & AC & C \\
\hline rs62459755 & 57008112 & G & 0.05 & GG & TG & G \\
\hline rs62459758 & 57008634 & G & 0.43 & GA & GA & A \\
\hline rs201707354 & 57008719 & C & 0.06 & CC & GC & C \\
\hline rs62460982 & 57011944 & C & 0.41 & CT & CT & T \\
\hline rs11981414 & 57188016 & A & 0.29 & AG & GG & G \\
\hline rs4577907 & 57195291 & C & 0.29 & CA & AA & A \\
\hline$\ldots \ldots$ & G... & & & & & \\
\hline rs60113999 & 64274517 & A & 0.32 & AG & AG & G \\
\hline rs7809431 & 64275284 & C & 0.98 & CC & TT & - \\
\hline indicates SNPs in this region share the same haplotype & & \\
\hline
\end{tabular}

\section{TRAP1}

In total, two novel frameshift variants (p.R13Cfs*107 and p.R449X) and two missense variants (p.E4A and p.R37W) in TRAP1 were identified in 4 patients (1.0\%). All the variants are located at the critical functional domain and classified as pathogenic/likely pathogenic or VUS. The stop-gain variant, R703X, which was located at the end of the $C$ terminus (Fig. 1C) and might not cause a functional change in the protein, was not included in further analysis. All four patients presented tremor as the initial symptom, good response to L-DOPA and a varied age of onset (ranging from 41.0-60.3 years). Three of them showed mild cognitive impairment (Table 2). The proband carrying p.R449X developed motor fluctuation and dyskinesia when the duration was less than 5 years. Interestingly, in this family, three consecutive generations including the proband's father, grandfather, the older brother of his father and the proband, had similar parkinsonian symptoms (Fig. 1H, family 6). However, cosegregation analysis could not be performed because DNA samples from his relatives were not available. 


\section{RHOT1}

Only one novel rare variant, p.S95N, in RHOT1 was found in a proband with the LOPD phenotype. The variant was the first mutation identified in the N-GTPase domain (Fig. 1D) and classified as likely pathogenic. The patient presented classical Parkinsonism and had a good response to L-DOPA. However, he developed mild cognitive dysfunction in the early stage of the disease (duration of 2.28 years). His mother presented right lower limb resting tremor at the age of 60 (Fig. 11, family 8).

\section{HSPA9}

Two novel missense variants, p.M70T and p.N180I, in HSPA9 were found in two probands with the EOPD phenotype. The two variants were located at the nucleotide binding domain (Fig. 1E) and classified as likely pathogenic. These two patients showed typical motor symptoms, a good response to L-DOPA and relatively slow disease progression (Table 2).

\section{Common variants in HTRA2 and CHCHD2 identified in ADPD}

Similar to previous studies on Asian PD patients, the common variant (p.G339S) in HTRA2 was not found in our cohort, though p.G339S was identified as a pathogenic variant and found in $0.5 \%$ of an European ancestry PD population. However, there was no difference in the frequency distribution of the variant p.G339S between patients and controls in both Asian and Caucasian populations based on a meta-analysis (Supplementary Table 4).

However, the p.P2L variant in $\mathrm{CHCHD2}$, which was reported to be exclusively associated with PD in Asian populations, was found in 7 ADPD patients in our cohort. Combined with previous studies, a meta-analysis revealed that the variant $\mathrm{p} . \mathrm{P} 2 \mathrm{~L}$ was associated with an increased risk for PD in Asian populations ( $\mathrm{P}$ $=4.7 \mathrm{e}-8, \mathrm{OR}=3.2,95 \% \mathrm{Cl}=2.1-5.0$ ) but not in Caucasian populations (Supplementary Table 4).

\section{Burden Analysis}

A gene-based burden test was performed to investigate whether rare PPVs in all six mitochondrial function-related PD genes collectively contribute to the risk for ADPD in the Chinese cohort (Table 3). Using the East Asian cohort in GnomAD v2.1 as a control, genes other than $\mathrm{CHCHD2} \mathrm{tended} \mathrm{to} \mathrm{be} \mathrm{slightly} \mathrm{enriched} \mathrm{in}$ ADPD patients $(p=0.05)$, and no rare PPVs in other genes were enriched in ADPD patients. In addition, no difference in the total frequency of rare PPVs between ADPD (1.1\%) and controls (1.0\%) was found.

Table 3

Summary of the gene-based burden test for rare putative pathogenic variants in ADPD

\begin{tabular}{|c|c|c|c|c|c|c|c|c|}
\hline \multirow[t]{2}{*}{ Gene } & \multicolumn{3}{|c|}{ Cases $(n=400)$} & \multicolumn{3}{|c|}{ Controls* $(n=9977)$} & \multirow[t]{2}{*}{ p-value } & \multirow[t]{2}{*}{ OR $(95 \% \mathrm{Cl})$} \\
\hline & $\%$ & PPV & NPPV & $\%$ & PPV & NPPV & & \\
\hline HTRA2 & 0.13 & 1 & 799 & 0.06 & 11 & 19943 & 0.376 & $2.27[0.29-17.60]$ \\
\hline CHCHD2 & 0.38 & 3 & 797 & 0.10 & 19 & 19935 & 0.051 & $3.95[1.17-13.37]$ \\
\hline $\mathrm{CHCHD10}$ & 0 & 0 & 800 & 0.05 & 9 & 19945 & 1.000 & - \\
\hline TRAP1 & 0.38 & 3 & 797 & 0.67 & 133 & 19821 & 0.316 & $0.56[0.18-1.77]$ \\
\hline RHOT1 & 0.13 & 1 & 799 & 0.09 & 17 & 19937 & 0.507 & $1.47[0.20-11.04]$ \\
\hline HSPA9 & 0.13 & 1 & 799 & 0.09 & 17 & 19937 & 0.507 & $1.47[0.20-11.04]$ \\
\hline Total & 1.13 & 9 & 791 & 1.03 & 206 & 19748 & 0.800 & $1.09[0.56-2.13]$ \\
\hline
\end{tabular}

PPV: putative pathogenic variants were predicted by by Condel;NPPV: non putative pathogenic variants $\square$ the putative pathogenic variants * rare variants data from East Asian cohort of GnomAD v2.1 which includes 9977 (19954 alleles) individuals, rare variants only include missense, splicing, stop gained, indel, start lost variants, excludes synonymy variants, intron variants.

\section{Discussion}

This was the first large study applying comprehensive genetic methods to explore the frequency of rare variants in six candidate Mendelian dominantly inherited mitochondrial function-associated genes and genotype-phenotype correlations in the ADPD population. The total frequency of patients with rare variants in our Chinese ADPD cohort was $3 \%(12 / 400)$, including $0.5 \%$ in HTRA2, $0.8 \%$ in CHCHD2, $1 \%$ in TRAP1, 0.3\% in RHOT1 and $0.5 \%$ in HSPA9. Overall, 12 rare variants with a frequency $\leq 0.1 \%$ were identified in the six genes; among them, 5 variants were protein-truncating variants, and 5 novel variants were identified.

For a long time, studies on environmental factors showed that exposure to insecticides, oxidants, and heavy metals increases the risk for individuals to develop PD, and studies on samples from blood, urine and cerebrospinal fluid or tissue from the postmortem brains of PD patients showed abnormal activity or levels of mitochondrial-related enzymes[17], suggesting that mitochondrial function impairment is important in the pathogenesis of the disease. With the advent of next-generation sequencing, a growing number of risk or causative genes have been identified in PD. However, genes other than parkin, PINK1 and DJ1 are mainly responsible for EOPD, and variants in other mitochondrial function-related genes are rarely reported, especially in ADPD. Much higher conservation of genetic sequences in functionally critical genes than in nonessential genes in evolutionary processes, such as the low frequency of rare PPVs shown in our burden analysis, could account for the rare mutations in these genes. 
HTRA2, which has four critical domains, including the mitochondrial targeting signal, transmembrane domain, trypsin-like domain, and PDZ domain, was first identified to be associated with PD in Germany[5]. The rare pathogenic variant (G399S) of HTRA2 was significantly enriched in PD (0.8\%, 4/518) and caused late-onset, typical parkinsonian symptoms[5]. Although the G399S variant was also found in another Caucasian cohort, its distribution between PD patients and controls was not different, thus raising questions on the pathogenic role of the variant in HTRA2 in PD[18-20]. Next, cosegregation analysis of a substantial essential tremor and PD in a family found that the affected individuals carried the same G399S variant[21]. Additionally, functional analysis of the variant based on experiments showed that the variant altered mitochondrial morphology and decreased protease activity[5]. Although G399S was not found in Asian PD patients, other rare variants of HTRA2 were identified in Asian individuals with PD[22, 23]. In our study, we found two ADPD patients with rare variants in HTRA2, and cosegregation analysis of one family supported its pathogenicity. In addition, as shown in Fig. 1A, most of the reported variants are located at the functional domain of HTRA2, suggesting that HTRA2 contributes to the development of ADPD.

CHCHD2 encodes a coiled-coil-helix-coiled-coil-helix domain-containing protein that is involved in the biogenesis and regulation of enzymes in the mitochondrial respiratory chain. Mutations in $C H C H D 2$ were first reported to cause autosomal dominant LOPD in Japanese people[6]. Later, several studies found that mutations in the $C H C H D 2$ gene are rare in $\mathrm{PD}$ among different ethnic populations, and less than fifteen rare variants of $\mathrm{CHCHD} 2$ were identified in PD to date (summarized in Fig. 1B and Supplementary Table 3). However, most of the reported variants are located at the mitochondrial targeting signal domain and/or $\mathrm{CHCH}$ domain, causing subcellular mislocalization and mitochondrial dysfunction[6]. In the current study, we found two truncated variants of the $\mathrm{CHCHD2}$ gene in 3 patients. Although the two variants, p.P53Afs*37 and p.Y99X, were also found in controls from East Asia, their frequencies in controls ( $1 / 9119$ and $1 / 9190$, respectively) were extremely low. Cosegregation analysis of one family confirmed that the affected individual carried p.P53Afs 37 exclusively, which partly supports its pathogenicity in PD. In addition, the finding that the p.P53Afs 37 variant may come from a common ancestor at least 65 generations ago suggested that it is not very rare in our cohort, which was further supported by the fact this variant was identified in a sporadic PD( $1 / 300$ ). Therefore, we recommended that the variant should be regularly screened in Chinese ADPD patients at least. Additionally, burden analysis found that rare PPVs tend to increase the collective risk for ADPD in Chinese patients, and the common variants p.P2L, a putative pathogenic variant, was confirmed to have an increased risk for PD exclusively in Asian populations[24] based on a meta-analysis including 6902 PD patients and 5999 controls. Hence, all evidence highlighted the important genetic role of $\mathrm{CHCHD} 2$ in PD.

HSPA9 is a member of the heat-shock cognate protein family, which plays a role in cell proliferation, stress response and maintenance of the mitochondria. Two pathogenic missense mutations in HSPA9 were first identified in two LOPD patients from Spain [9, 11]. Then, another missense variant, A476T in HSPA9, was identified in 5 LOPD patients and 6 controls, and further functional analysis confirmed that variants in the gene cause loss of function and mitochondrial function impairment. Interestingly, four of these six controls with the A476T variant, who were significantly older than the other two asymptomatic carriers, were reported to have defined extrapyramidal symptoms but did not fulfill the diagnosis of PD, suggesting that variants in HSPA9 might be associated with incomplete penetrance for PD[25], which could also account for why rare variants in HSPA9 were not enriched in EOPD [26]. However, in our study, two likely pathogenic variants, p.M70T and p.N180I in HSPA9 were identified in two early onset ADPD patients. Different distributions of variants in the functional domain of HSPA9, epigenetics or environmental factors, could be responsible for the discrepancy in age of onset.

Recently, there was a focus on the mitochondrial chaperone protein TRAP1 in the field of PD studies because it mitigates a-synuclein toxicity[27] and rescues the PINK1 loss-of-function phenotype[8]. A truncated mutation, p.R47X in TRAP1, which leads to reduced control of energy metabolism, impacting mitochondrial membrane potential, was found in an LOPD patient[10]. However, no pathogenic variants were found in a study on 181 PD patients from another Caucasian cohort [28] to against the pathogenic role of this gene in PD. However, in our ADPD cohort, four rare variants, including two frameshift mutations that caused a truncated protein and two missense mutations, were first identified in Asia and predicted to be deleterious, which provided more evidence that TRAP1 is involved in mitochondrial dysfunction in PD.

Very recently, four rare missense variants in RHOT1 were identified in PD patients from Caucasian populations. Using patient-derived fibroblasts, the researchers observed that these variants impaired calcium homeostasis and caused alterations of ER-mitochondria contact sites, ultimately affecting energy metabolism and increasing mitophagy $[12,13]$. The likely pathogenic variant identified in our study, p.S95N, is located in the N-GTPase domain, which is different from the location of the four reported variants. Therefore, the variant may provide an additional genetic model to explore a new mechanism for RHOT1 function in PD.

To date, the limited number of PD patients with mitochondrial function-related gene variants reported in previous studies makes it difficult to characterize the clinical phenotype for PD caused by mutations of these genes. Our study identified 12 patients with rare variants in the six genes in Chinese ADPD, which makes it more comprehensive and accurate to sum phenotypes for each gene or gene complex. Although late-onset age is usually suggested in autosomal dominantly inherited monogenic PD[29], rare variants were identified in our EOPD patients, such as 2 patients carrying $\mathrm{CHCHD2}$ variants, 2 patients carrying TRAP1 variants and 2 patients carrying HSPA9. In accord with previous studies, mutations in the six genes cause PD with varied age of onset, indicating that genetic screening for the six genes in EOPD patients should not be overlooked. In addition, although the different roles of the six genes in mitochondrial function can be classified at different levels-molecular (HTRA2, TRAP1 and HSPA9), organellar (RHOT1) and cellular (CHCHD2 and CHCHD10)-the dysfunction of mitochondrial quality control machinery is the same pathway involved in the pathogenicity of PD[30], suggesting that patients with rare variants in the six genes might share a similar phenotype. Similar to the hypothesis, all of our patients carrying rare variants in the genes presented typical motor symptoms and good response to L-DOPA, and most of them showed slower disease progression (8/12, UPDRS-III/duration ratio less than 10) and mild cognitive impairment $(9 / 12)(\mathrm{MOCA}<26)$, even at the early stage of these diseases. Therefore, our study expands the PD phenotype for patients with mutations in these genes.

Although burden analysis in our study argued against a significant overrepresentation of PPVs for each gene or the six genes together in ADPD patients, the results reported in some previous studies indicated no differences in frequency distribution for rare variants in some mitochondrial function-related genes between PD and controls, such as HTRA2[19], HSPA9[26] and TRAP1[28]; thus, all the results should warrant cautious interpretation. In our burden analysis,

Page $7 / 11$ 
the consensus deleteriousness score and Condel were used to help exclude rare but benign missense variants in PD and the controls from the database; however, the pathogenicity that was predicted to be a deleterious variant should be further confirmed based on more experimental evidence. The analysis using the data from the east Asian GnomAD database rather than ethnically age- and sex-matched controls may also contribute such negative findings. In addition, highly conserved genes, etiological heterogeneity, incomplete penetrance and late-onset diseases make it difficult to detect associations between rare variants and the disease in case-control studies. In addition, the methods of variant detection and age of onset varied among the different studies, and public databases also caused deviation. Overall, we do not deny the role of the candidate mitochondrial genes involved in PD.

\section{Conclusion}

Overall, our study identified 12 rare variants in six controversial mitochondrial function-related PD genes, which accounted for $3 \%$ of Chinese ADPD cases and provided more evidence of mitochondrial dysfunction involved in the pathogenicity of PD. Among them, five novel variants expanded the mutation spectra and provided more genetic variant selection in mechanistic research for the genes. Based on the data from the common founder analysis and further scanning for p.P53Afs $* 37$ in sporadic cases, burden analysis for rare variants, meta-analysis for common risk variants, the $\mathrm{CHCHD} 2 \mathrm{gene}$ should be given more attention in PD originating from the Chinese population. In addition, genotype-phenotype analysis enhances the understanding of the PD phenotype in mitochondrial function-related gene variant carriers.

\section{Abbreviations}

PD: Parkinson's disease; ADPD: autosomal dominant Parkinson's disease; ALS: amyotrophic lateral sclerosis; EOPD: early-onset PD; FPD: familial PD; WES: Whole Exome Sequencing; PARK2: parkin; PINK1: PTEN-induced putative kinase 1; HTRA2: high-temperature-regulated A2; ; CHCHD2: coiled-coil-helix-coiledcoil-helix domain containing 2; CHCHD10: coiled-coil-helix-coiled-coil-helix domain containing 10; TRAP1: tumor necrosis factor receptor associated protein-1; HSPA9: heat shock 70 kDa protein 9; RHOT1: ras homolog family member T1; ER: endoplasmic reticulum; UPDRS: Unified Parkinson's Disease Rating Scale; MoCA: Montreal Cognitive Assessment; FAB: frontal assessment battery; ACMG: American College of Medical Genetics and Genomics; PPVs: putative pathogenic variants

\section{Declarations}

\section{Ethics approval and consent to participate:}

Approval was obtained from the Ethics Committee of West China Hospital of SichuanUniversity. Informed consent was obtained from all subjects.

\section{Consent for publish:}

All participants were properly consented for this study.

\section{Availability of data and materials:}

All data supporting our findings are available from the corresponding author on reasonable request.

\section{Competing interests:}

The authors declare no conflict of interest.

\section{Funding:}

This study was supported the National Key Research and Development Program of China (grant no. 2016YFC0901504), the National Natural Science Fund of China (Grant No. 81571247) and the 1.3.5 project for disciplines of excellence, West China Hospital, Sichuan University (Grant No. ZYJC18038 and Grant No. ZYJC18003).

\section{Authors' contributions:}

Yongping Chen: Design, Execution, data analysis and Writing

Xiaojing Gu: Execution, Blood samples collection and DNA extraction

Ruwei Ou: Execution, Blood samples collection and clinical data collection

Lingyu Zhang: Execution, Blood samples collection and clinical data collection

Yanbing Hou: Execution, clinical data collection

Kuncheng Liu: Execution, clinical data collection

Bei Cao: Execution, patients' enrollment and clinical data collection

Qianqian Wei: Execution, clinical data collection 
Wei Song: Execution, patients' enrollment

Bi Zhao: Execution, patients' enrollment

Ying Wu: Execution, patients' enrollment

Jingqiu Cheng: Review and Critique

Huifang Shang: Conception, Design, Organization, Review and Critique

All authors read and approved the final manuscript.

\section{Acknowledgements:}

The authors appreciate all cohort individuals and their families for their participation in this study.

\section{Authors' information:}

Huifang Shang, Neurologist, Vice director in the Department of Neurology, West China Hospital, Sichuan University.

\section{References}

1. de Lau LM, Breteler MM. Epidemiology of Parkinson's disease. Lancet Neurol. 2006;5:525-35.

2. Mann VM, Cooper JM, Krige D, Daniel SE, Schapira AH, Marsden CD. Brain, skeletal muscle and platelet homogenate mitochondrial function in Parkinson's disease. Brain. 1992;115(Pt 2):333-42.

3. Parker WD Jr, Parks JK, Swerdlow RH. Complex I deficiency in Parkinson's disease frontal cortex. Brain Res. 2008;1189:215-8.

4. Lunati A, Lesage S, Brice A. The genetic landscape of Parkinson's disease. Rev Neurol (Paris). 2018;174:628-43.

5. Strauss KM, Martins LM, Plun-Favreau H, Marx FP, Kautzmann S, Berg D, Gasser T, Wszolek Z, Muller T, Bornemann A, et al. Loss of function mutations in the gene encoding Omi/HtrA2 in Parkinson's disease. Hum Mol Genet. 2005;14:2099-111.

6. Funayama M, Ohe K, Amo T, Furuya N, Yamaguchi J, Saiki S, Li Y, Ogaki K, Ando M, Yoshino H, et al. CHCHD2 mutations in autosomal dominant lateonset Parkinson's disease: a genome-wide linkage and sequencing study. Lancet Neurol. 2015;14:274-82.

7. Bannwarth S, Ait-El-Mkadem S, Chaussenot A, Genin EC, Lacas-Gervais S, Fragaki K, Berg-Alonso L, Kageyama Y, Serre V, Moore DG, et al. A mitochondrial origin for frontotemporal dementia and amyotrophic lateral sclerosis through CHCHD10 involvement. Brain. 2014;137:2329-45.

8. Zhang L, Karsten P, Hamm S, Pogson JH, Muller-Rischart AK, Exner N, Haass C, Whitworth AJ, Winklhofer KF, Schulz JB, Voigt A. TRAP1 rescues PINK1 loss-of-function phenotypes. Hum Mol Genet. 2013;22:2829-41.

9. Wadhwa R, Ryu J, Ahn HM, Saxena N, Chaudhary A, Yun CO, Kaul SC. Functional significance of point mutations in stress chaperone mortalin and their relevance to Parkinson disease. J Biol Chem. 2015;290:8447-56.

10. Fitzgerald JC, Zimprich A, Carvajal Berrio DA, Schindler KM, Maurer B, Schulte C, Bus C, Hauser AK, Kubler M, Lewin R, et al. Metformin reverses TRAP1 mutation-associated alterations in mitochondrial function in Parkinson's disease. Brain. 2017;140:2444-59.

11. De Mena L, Coto E, Sanchez-Ferrero E, Ribacoba R, Guisasola LM, Salvador C, Blazquez M, Alvarez V. Mutational screening of the mortalin gene (HSPA9) in Parkinson's disease. J Neural Transm (Vienna). 2009;116:1289-93.

12. Grossmann D, Berenguer-Escuder C, Bellet ME, Scheibner D, Bohler J, Massart F, Rapaport D, Skupin A, Fouquier d'Herouel A, Sharma M, et al. Mutations in RHOT1 Disrupt Endoplasmic Reticulum-Mitochondria Contact Sites Interfering with Calcium Homeostasis and Mitochondrial Dynamics in Parkinson's Disease. Antioxid Redox Signal. 2019;31:1213-34.

13. Berenguer-Escuder C, Grossmann D, Massart F, Antony P, Burbulla LF, Glaab E, Imhoff S, Trinh J, Seibler P, Grunewald A, Kruger R. Variants in Miro1 Cause Alterations of ER-Mitochondria Contact Sites in Fibroblasts from Parkinson's Disease Patients. J Clin Med $2019,8$.

14. Hughes AJ, Daniel SE, Kilford L, Lees AJ. Accuracy of clinical diagnosis of idiopathic Parkinson's disease: a clinico-pathological study of 100 cases. J Neurol Neurosurg Psychiatry. 1992;55:181-4.

15. Postuma RB, Berg D, Stern M, Poewe W, Olanow CW, Oertel W, Obeso J, Marek K, Litvan I, Lang AE, et al. MDS clinical diagnostic criteria for Parkinson's disease. Mov Disord. 2015;30:1591-601.

16. Puschmann A. Monogenic Parkinson's disease and parkinsonism: clinical phenotypes and frequencies of known mutations. Parkinsonism Relat Disord. 2013;19:407-15.

17. Park JS, Davis RL, Sue CM. Mitochondrial Dysfunction in Parkinson's Disease: New Mechanistic Insights and Therapeutic Perspectives. Curr Neurol Neurosci Rep. 2018;18:21.

18. Ross OA, Soto Al, Vilarino-Guell C, Heckman MG, DiehI NN, Hulihan MM, Aasly JO, Sando S, Gibson JM, Lynch T, et al. Genetic variation of Omi/HtrA2 and Parkinson's disease. Parkinsonism Relat Disord. 2008;14:539-43.

19. Simon-Sanchez J, Singleton AB. Sequencing analysis of OMI/HTRA2 shows previously reported pathogenic mutations in neurologically normal controls. Hum Mol Genet. 2008;17:1988-93.

20. Kruger R, Sharma M, Riess O, Gasser T, Van Broeckhoven C, Theuns J, Aasly J, Annesi G, Bentivoglio AR, Brice A, et al. A large-scale genetic association study to evaluate the contribution of Omi/HtrA2 (PARK13) to Parkinson's disease. Neurobiol Aging. 2011;32:548 e549-518.

Page 9/11 
21. Unal Gulsuner H, Gulsuner S, Mercan FN, Onat OE, Walsh T, Shahin H, Lee MK, Dogu O, Kansu T, Topaloglu H, et al. Mitochondrial serine protease HTRA2 p.G399S in a kindred with essential tremor and Parkinson disease. Proc Natl Acad Sci U S A. 2014;111:18285-90.

22. Lin CH, Chen ML, Chen GS, Tai CH, Wu RM. Novel variant Pro143Ala in HTRA2 contributes to Parkinson's disease by inducing hyperphosphorylation of HTRA2 protein in mitochondria. Hum Genet. 2011;130:817-27.

23. Chen $\mathrm{CM}$, Wu CH, Hsieh CH, Lin CH, Chen IC, Chen YC, Lee LC, Lee CM, Tseng YC, Lee-Chen GJ, Wu YR. HTRA2 variations in Taiwanese Parkinson's disease. J Neural Transm (Vienna). 2014;121:491-8.

24. Yang X, Zhao Q, An R, Zheng J, Tian S, Chen Y, Xu Y. Mutational scanning of the CHCHD2 gene in Han Chinese patients with Parkinson's disease and meta-analysis of the literature. Parkinsonism Relat Disord. 2016;29:42-6.

25. Burbulla LF, Schelling C, Kato H, Rapaport D, Woitalla D, Schiesling C, Schulte C, Sharma M, Illig T, Bauer P, et al. Dissecting the role of the mitochondrial chaperone mortalin in Parkinson's disease: functional impact of disease-related variants on mitochondrial homeostasis. Hum Mol Genet. 2010;19:443752.

26. Freimann K, Zschiedrich K, Bruggemann N, Grunewald A, Pawlack H, Hagenah J, Lohmann K, Klein C, Westenberger A. Mortalin mutations are not a frequent cause of early-onset Parkinson disease. Neurobiol Aging. 2013;34:2694 e2619-20.

27. Butler EK, Voigt A, Lutz AK, Toegel JP, Gerhardt E, Karsten P, Falkenburger B, Reinartz A, Winklhofer KF, Schulz JB. The mitochondrial chaperone protein TRAP1 mitigates alpha-Synuclein toxicity. PLoS Genet. 2012;8:e1002488.

28. Gaare JJ, Nido GS, Sztromwasser P, Knappskog PM, Dahl O, Lund-Johansen M, Alves G, Tysnes OB, Johansson S, Haugarvoll K, Tzoulis C. No evidence for rare TRAP1 mutations influencing the risk of idiopathic Parkinson's disease. Brain. 2018;141:e16.

29. Pang SY, Ho PW, Liu HF, Leung CT, Li L, Chang EES, Ramsden DB, Ho SL. The interplay of aging, genetics and environmental factors in the pathogenesis of Parkinson's disease. Transl Neurodegener. 2019;8:23.

30. Larsen SB, Hanss Z, Kruger R. The genetic architecture of mitochondrial dysfunction in Parkinson's disease. Cell Tissue Res. 2018;373:21-37.

\section{Figures}
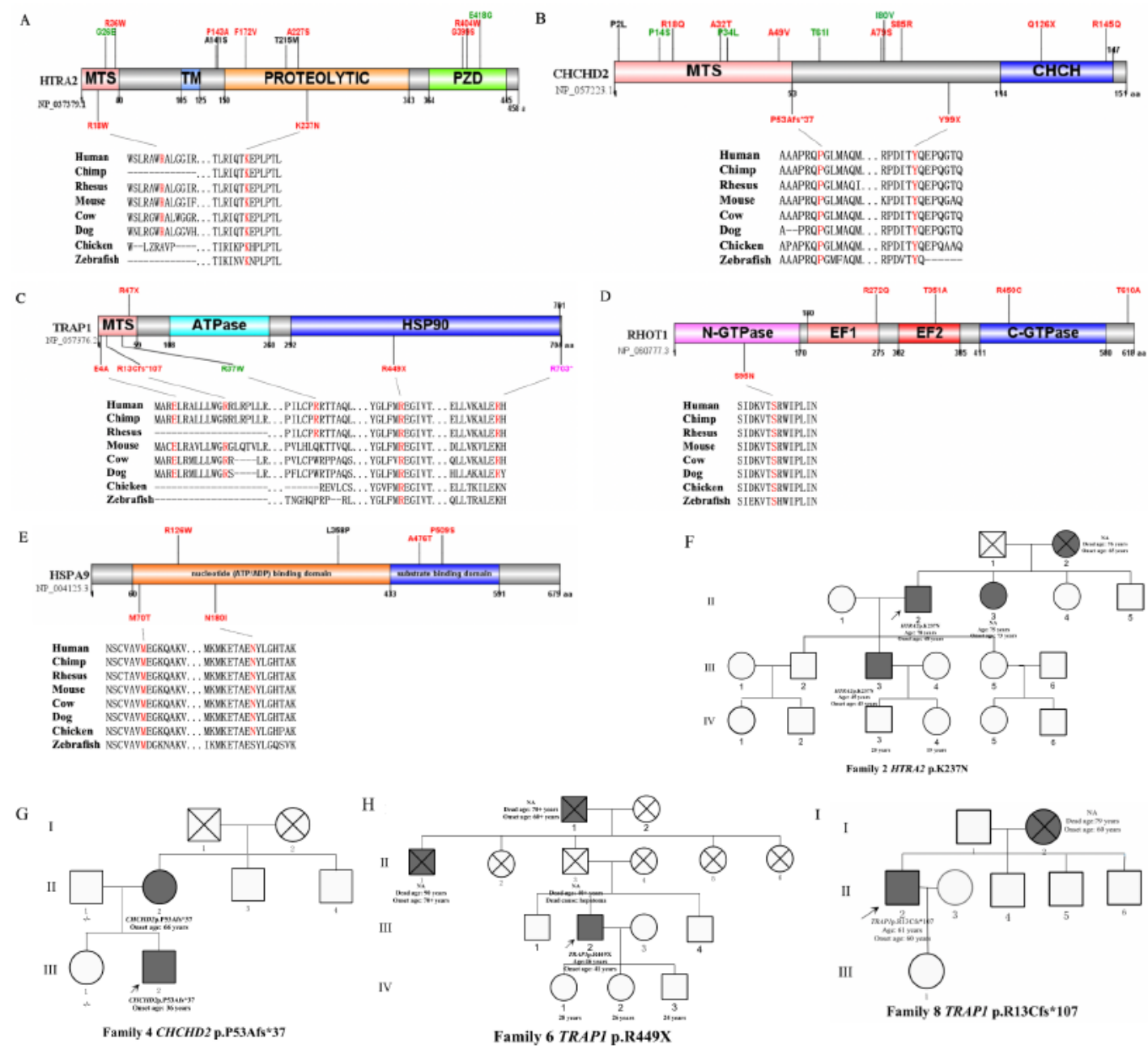

Figure 1 
Variants identified in the schematic representations and protein sequence alignment analysis of these five mitochondrialrelated genes, and pedigree chart of special patients with gene mutations in this study. (A-E) The variants on the upper of schematic representations were reported in previous studies, the variants under the schematic representations were identified in this study. MTS: mitochondrial targeting signal, $\mathrm{CHCH}$ : coiled coil I-helix I-coiled coil 2-helix 2, Red denotes pathogenic/likely pathogenic variants, Green denotes uncertain significance (US) variants, pink denotes benign, black denotes polymorphisms. $A$, Domain organization of hwnan HtrA2 protein. TM: trans-membrane domain, PROTEOLYTIC: trypsin-like domain, PDZ: PDZ domain; B, Domain organization of human CHCHD2 protein; C, Domain organization of human TRAP I protein; D, Domain organization of human RHOTI protein. Cartoon showing the domain structure of Miro proteins, with the N-terminal GTPase domain, EF hands, C-terminal GTPase domain and C-terminal membrane anchor depicted as cylinders; E, Domain organization of human HSPA9 protein. (F-1) Pedigree chart of patients with gene mutations, Black symbols denote PD patients, oblique arrow indicate the probands, fork symbols indicate deceased individuals, circles indicate women and squares indicate men,"-" indicates the wildtype allele, NA indicates the DNA was not available.

\section{Supplementary Files}

This is a list of supplementary files associated with this preprint. Click to download.

- 3SupplementaryTables.docx

- supplymentarymethods.docx

- 3SupplementaryTables.docx

- supplymentarymethods.docx 